\title{
Simplified Ink Spreading Equations for CMYK Halftone Prints
}

\author{
Thomas Bugnon, Mathieu Brichon and Roger David Hersch \\ École Polytechnique Fédérale de Lausanne (EPFL), \\ School of Computer and Communication Sciences, Lausanne 1015, Switzerland
}

\begin{abstract}
The Yule-Nielsen modified spectral Neugebauer model enables predicting reflectance spectra from surface coverages. In order to provide high prediction accuracy, this model is enhanced with an ink spreading model accounting for physical dot gain. Traditionally, physical dot gain, also called mechanical dot gain, is modeled by one ink spreading curve per ink. An ink spreading curve represents the mapping between nominal to effective dot surface coverages when an ink halftone wedge is printed. In previous publications, we have shown that using one ink spreading curve per ink is not sufficient to accurately model physical dot gain, and that the physical dot gain of a specific ink is modified by the presence of other inks. We therefore proposed an ink spreading model taking all the ink superposition conditions into account. We now show that not all superposition conditions are useful and necessary when working with cyan, magenta, yellow, and black inks. We therefore study the influence of ink spreading in different superposition conditions on the accuracy of the spectral prediction model. Finally, we propose new, simplified ink spreading equations that better suit CMYK prints and are more resilient to noise.
\end{abstract}

Keywords: color reproduction, ink spreading model, spectral prediction model, Yule-Nielsen modified Spectral Neugebauer, halftones, ink spreading curves, dot gain, ink superposition conditions, noise resilience

\section{INTRODUCTION}

The goal of a color reproduction system is to be able to reproduce input colors as accurately as possible. This is not a trivial task since the human visual system is very sensitive to small color differences. In printing systems, there are many factors influencing the range of printable colors: the inks, the substrate (paper, plastic, glass, etc.), the illumination conditions, and the halftones. Spectral reflection prediction models are helpful in studying the influence of these factors.

One of the fundamental aspects a spectral reflection prediction model has to consider is how the inks spread on the paper, a phenomenon also referred to as physical or mechanical dot gain. With an ink spreading model accounting for physical dot gain, a spectral reflection prediction model is able to accurately predict reflectance spectra in function of ink surface coverages for three or four inks ${ }^{1,2,3}$. In order to be effective, such an ink spreading model must take into account not only the interaction between an ink halftone and paper, but also the interaction between an ink halftone and superposed inks.

One proposed solution is to use multiple ink spreading curves, also called tone reproduction curves, to characterize the physical dot gain of the ink halftones on paper in all solid ink superposition conditions. For CMYK halftone prints, such an approach requires the characterization of $32 \mathrm{ink}$ spreading curves. The aim of this paper is to evaluate the relevance of each ink spreading curve and subsequently simplify the ink spreading model in order to use fewer curves. The relevance of an ink spreading curve depends on its impact on the spectral prediction model accuracy as well as how it is affected by noise.

Section 2 introduces the Yule-Nielsen modified Spectral Neugebauer model that is used as spectral reflection prediction model. The ink spreading model and the characterization of the ink spreading curves are detailed in Section 3 . Section 4 evaluates the influence of each ink spreading curve on the accuracy of the prediction model and Section 5 the influence of measurement noise on the characterization of the ink spreading curves. In Section 6, a new set of equations that reduces the number of component equations of the ink spreading model is proposed and evaluated against the existing set of equations. We finally draw the conclusions in Section 7. 


\section{SPECTRAL PREDICTION MODELS AND RELATED APPROACHES}

Many different phenomena influence the reflection spectrum of a color halftone patch printed on a diffusely reflecting substrate (e.g. paper). These phenomena comprise the surface (Fresnel) reflection at the interface between the air and the paper, light scattering and reflection within the substrate (i.e. the paper bulk), and the internal (Fresnel) reflections at the interface between the paper and the air.

The lateral scattering of light within the paper substrate and the internal reflections at the interface between the paper and the air are responsible for what is generally called optical dot gain, also known as the Yule-Nielsen effect. In addition, due to the printing process, deposited ink surface coverages are generally larger than nominal coverages, yielding a physical dot gain also referred to as mechanical dot gain. Such effective ink surface coverages depend on the inks, the paper, and also the specific ink superposition conditions, i.e. the superposition of ink halftones and solid inks.

At the present time and according to the literature ${ }^{4,5,6}$, mainly the well-known Yule-Nielsen modified Spectral Neugebauer model ${ }^{7,8}$ seems to be used in practice.

\subsection{The Yule-Nielsen modified Spectral Neugebauer model (YNSN)}

One of the first spectral models is the Neugebauer model ${ }^{9}$. In its original form, it predicts the CIE-XYZ tri-stimulus values of a color halftone patch as the sum of the tri-stimulus values of their individual colorants weighted by their fractional area coverages $a_{i}$. By considering the reflection spectra $R_{i}$ of colorants instead of their respective tri-stimulus values, one obtains the spectral Neugebauer equations ${ }^{8}$. They predict the reflection spectrum of a printed color halftone patch as a function of the reflection spectra of its individual colorants (also called Neugebauer primaries):

$$
R(\lambda)=\sum_{i} a_{i}^{*} R_{i}(\lambda)
$$

With $k$ inks, there are $2^{k}$ colorants: white, the $k$ single ink colorants and all the different superpositions of solid inks. For example, the red colorant is the superposition of the magenta and yellow inks. When the ink layers are printed independently one from another, the fractional area coverages of the individual colorants are closely approximated from the ink surface coverages by the Demichel equations ${ }^{10}$. These equations are shown in Figure 1 for the case of 2 inks, but can be extended to accommodate any number of inks ${ }^{4}$.

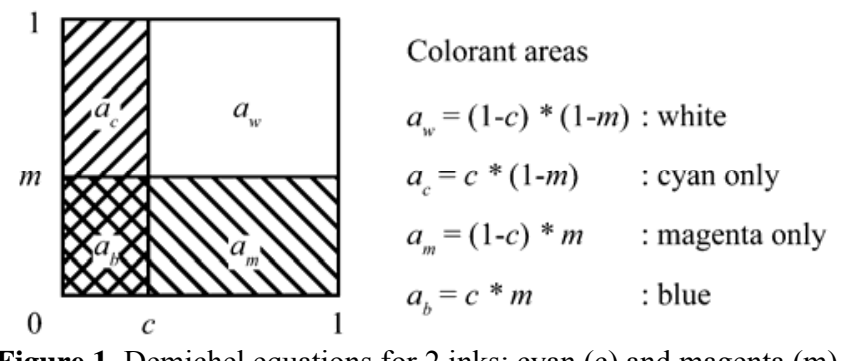

Figure 1. Demichel equations for 2 inks: cyan (c) and magenta (m).

The Neugebauer model is a generalization of the Murray-Davis model ${ }^{11}$ whose colorants are formed by only one ink and the paper white. Since the Neugebauer model neither takes explicitly into account the lateral propagation of light within the paper bulk nor the internal reflections (Fresnel reflections) at the paper-air interface, its predictions are not accurate ${ }^{12}$. Yule and Nielsen ${ }^{7}$ modeled the non-linear relationship between the reflectance of paper, single ink halftones, and solid inks by a power function whose exponent $n$ can be optimized according to the reflectance of a limited set of color patches. Viggiano ${ }^{8}$ applied the Yule-Nielsen relationship to the spectral Neugebauer equations, yielding the YuleNielsen modified Spectral Neugebauer model (YNSN):

$$
R(\lambda)=\left(\sum_{i} a_{i} * R_{i}(\lambda)^{\frac{1}{n}}\right)^{n}
$$

This YNSN model has been used by many researchers for the characterization of printing systems $5,8,12,13,14,15,16,17$. This model therefore plays a significant role in building color management systems. 


\subsection{The Ink Spreading Enhanced Yule-Nielsen modified Spectral Neugebauer model (EYNSN)}

One of the latest models is the Yule-Nielsen modified Spectral Neugebauer model enhanced with an ink spreading model $(\mathrm{EYNSN})^{1,2}$. The ink spreading model is used to account for physical dot gain, i.e. the fact that the inks spread out on the paper, resulting in effective ink surface coverages usually larger than the intended nominal ink surface coverages.

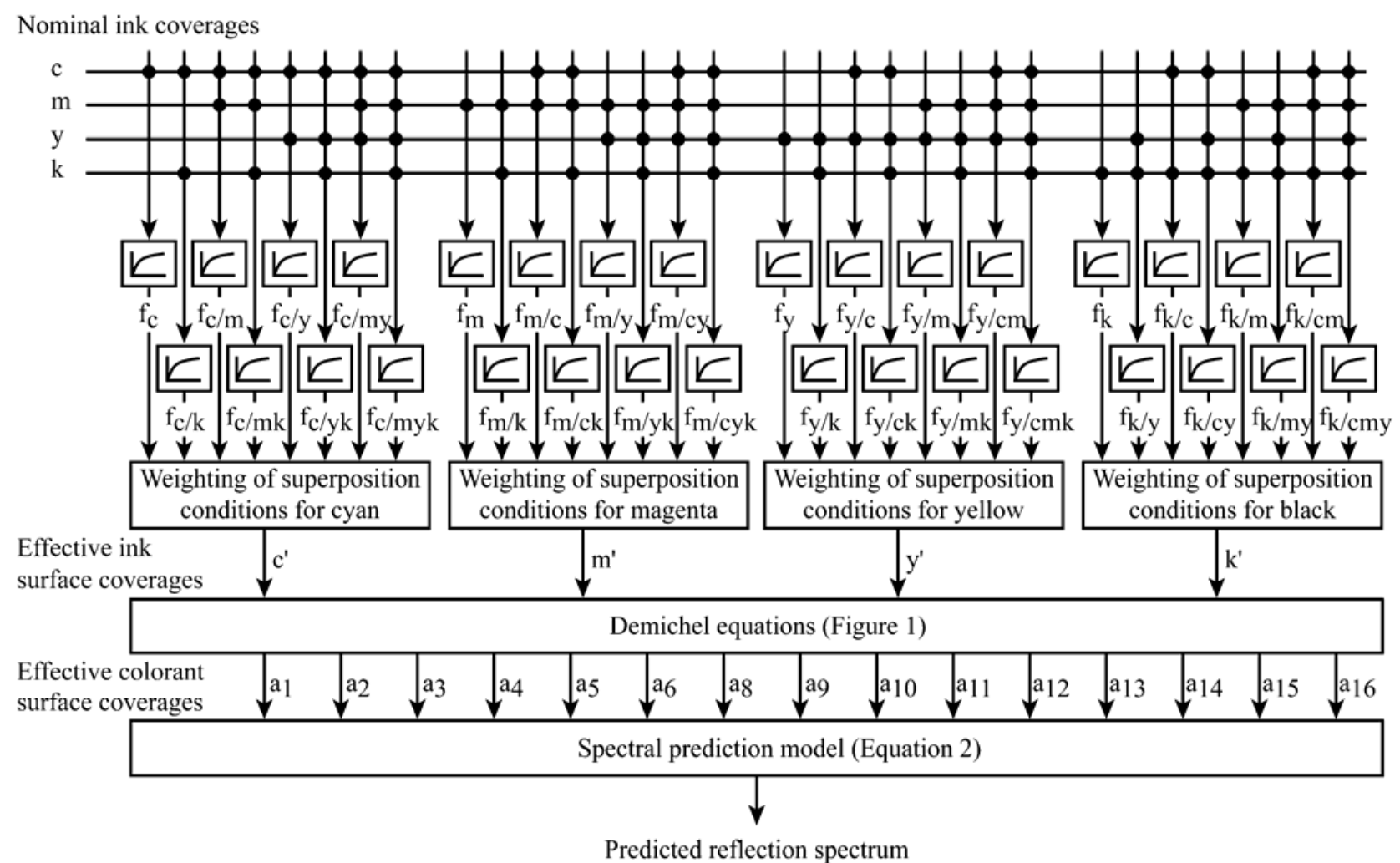

Figure 2. The Ink Spreading enhanced Yule-Nielsen modified Spectral Neugebauer model with nominal ink surface coverages $c, m, y$, and $k$; effective single ink halftone surface coverage of ink $i$ superposed with solid inks $j$ and $k f_{i j k}$; effective ink surface coverages $c^{\prime}$, $m^{\prime}, y^{\prime}$, and $k^{\prime}$; and effective colorant surface coverages $a_{1}$ to $a_{16}$.

Spectral predictions using the EYNSN are performed according to Figure 2. First, the effective single ink halftone surface coverages for the contributing superposition conditions are determined from nominal surface coverages by using the tone reproduction curves established during model calibration. There is one curve for each ink halftone in each superposition condition, i.e. an ink halftone superposed with paper only, and with one, two, or three given solid inks. In order to obtain the effective surface coverages of the inks forming a color halftone, the previously stored effective single ink halftone surface coverages are weighted according to the surface coverages of the colorants contributing to that color halftone. With the Demichel equations, we can then compute the corresponding effective surface coverages of the colorants forming that color halftone. Finally, from these effective colorant coverages, the YNSN model computes the predicted reflection spectrum.

Note that it is possible to replace the Yule-Nielsen modified Spectral Neugebauer model (Equation 2) by a Clapper-Yule model $^{1}$ extended to account for middle and low screen frequencies.

\section{THE INK SPREADING MODEL AND ITS DIRECTIVES}

In contrast to ink spreading models proposed in the literature, the proposed ink spreading model does not consider the dot gain of each ink separately, but takes the ink superposition conditions into consideration. The amount of dot gain of an ink depends on whether the ink halftone is printed alone on paper or in superposition with one or more other inks.

The influence of the superposition with other inks is modeled by using more than one ink spreading curve per ink. The effective ink surface coverages are then computed using a set of equations weighting the mapping performed by the ink 
spreading curves. There are different possible sets of equations depending on the assumptions made on how the inks influence each other. Each set of equation is further referred to as an ink spreading directive used by the ink spreading model. We first present the available ink spreading curves and how they are created during the calibration of the spectral prediction model. We then describe three different ink spreading directives.

\subsection{Available ink spreading curves and their calibration}

An ink spreading curve maps the nominal surface coverages of an ink into its effective surface coverages, i.e. to the surface that the ink effectively covers once printed on paper. Using the proposed ink spreading model, the amount by which an ink spreads out depends on the presence of other superposed inks. There are therefore several ink spreading curves for each ink halftone. For example, cyan may be printed alone, superposed to solid magenta, or superposed to solid yellow and solid black. In total, for four inks, there are 8 different superposition conditions for each ink, yielding 8 different ink spreading curves for each ink and a total of 32 ink spreading curves. The possible ink spreading curves are listed in Table 1.

Note that the ink spreading curves used for calibrating the spectral prediction model are defined for superposition conditions composed of solid inks only. There is for example a cyan ink spreading curve over solid magenta, but no cyan ink spreading curve over $50 \%$ magenta.

The following notation is used for the ink spreading curves: ink halftone/superposed inks. For example, $c / m$ refers to the cyan halftone over solid magenta ink spreading curve, $c$ my refers to cyan over solid magenta and solid yellow, and $c$ refers to the ink spreading curve of a cyan halftone printed alone.

These ink spreading curves can be seen as functions that take a nominal coverage as input and return the corresponding effective coverage as output. Within equations, the letter $f$ identifies such a function. For example, the function corresponding to the cyan over magenta ink spreading curve is $f_{c / m}(c)$, where $c$ is the cyan nominal coverage.

Table 1. List of the available ink spreading curves

\begin{tabular}{|l|l|l|l|l|l|l|l|}
\hline \multicolumn{2}{|c|}{ Cyan } & \multicolumn{2}{c|}{ Magenta } & \multicolumn{2}{c|}{ Yellow } & \multicolumn{2}{c|}{ Black } \\
\hline $\mathrm{c}$ & $\mathrm{c} / \mathrm{k}$ & $\mathrm{m}$ & $\mathrm{m} / \mathrm{k}$ & $\mathrm{y}$ & $\mathrm{y} / \mathrm{k}$ & $\mathrm{k}$ & $\mathrm{k} / \mathrm{y}$ \\
\hline $\mathrm{c} / \mathrm{m}$ & $\mathrm{c} / \mathrm{mk}$ & $\mathrm{m} / \mathrm{c}$ & $\mathrm{m} / \mathrm{ck}$ & $\mathrm{y} / \mathrm{c}$ & $\mathrm{y} / \mathrm{ck}$ & $\mathrm{k} / \mathrm{c}$ & $\mathrm{k} / \mathrm{cy}$ \\
\hline $\mathrm{c} / \mathrm{y}$ & $\mathrm{c} / \mathrm{yk}$ & $\mathrm{m} / \mathrm{y}$ & $\mathrm{m} / \mathrm{yk}$ & $\mathrm{y} / \mathrm{m}$ & $\mathrm{y} / \mathrm{mk}$ & $\mathrm{k} / \mathrm{m}$ & $\mathrm{k} / \mathrm{my}$ \\
\hline $\mathrm{c} / \mathrm{my}$ & $\mathrm{c} / \mathrm{myk}$ & $\mathrm{m} / \mathrm{cy}$ & $\mathrm{m} / \mathrm{cyk}$ & $\mathrm{y} / \mathrm{cm}$ & $\mathrm{y} / \mathrm{cmk}$ & $\mathrm{k} / \mathrm{cm}$ & $\mathrm{k} / \mathrm{cmy}$ \\
\hline
\end{tabular}

An ink spreading curve characterizes how a given ink halftone behaves in a given superposition condition, i.e. it characterizes the color wedge printed with a given ink halftone varying between $0 \%$ and $100 \%$ surface coverage superposed with given solid inks.

Such an ink spreading curve is calibrated with spectral measurements of one or more color patches forming that wedge. The spectral measurements must span the visible wavelength range $(380-730 \mathrm{~nm})$, and may be extended to the infrared wavelength range $(730-850 \mathrm{~nm})^{3}$. In this paper, we use spectral measurements comprising both the visible and the infrared wavelength ranges (380-850 nm). The black ink is pigment-based and absorbs light in the near infrared range. The other inks are dye-based and do not absorb in the near infrared range. For each measured patch, we find the effective surface coverage that minimizes the sum of square differences between the measured spectrum and the spectrum predicted by the YNSN model. Each measured patch provides one point of the ink spreading curve, i.e. it maps a given nominal surface coverage to an effective surface coverage. The entire function is computed by interpolating linearly between the available points.

Each ink spreading curve is independent from the other ink spreading curves and can be calibrated using a different number of patches. In the present work, we calibrate all the ink spreading curves using a single patch whose nominal surface coverage is $50 \%$. We therefore require 32 patches to calibrate all the ink spreading curves.

\subsection{Ink spreading directives}

When using more than one ink spreading curve per ink, there are many possible combinations to compute the effective ink coverages. Each directive of the ink spreading model specifies a different combination based on certain assumptions. Below, we describe the three directives presented by Hersch et. al. ${ }^{1,2}$. 
In the following equations, the cyan, magenta, yellow, and black nominal coverages are designated using the variables $c$, $m, y$, and $k$, respectively, whereas the effective coverages are designated using the variables $c^{\prime}, m^{\prime}, y^{\prime}$, and $k^{\prime}$.

\section{a) Single ink dot gain}

In the literature, ink spreading is often characterized by one ink spreading curve per ink and does not take superposition conditions into account ${ }^{5}$. In that case, the effective coverage of an ink depends only on the nominal coverage of the ink and is computed from the ink spreading curve of the ink alone. This ink spreading directive is further referred to as the single directive and is expressed by the following equations:

$$
c=f_{c}(c) \quad m=f_{m}(m) \quad y=f_{y}(y) \quad k=f_{k}(k)
$$

The problem with the single directive is that it does not take superposition conditions into consideration.

\section{b) Ink spreading of an ink halftone printed on top of ink layers}

The next directive, further referred to as the top directive, assumes that an ink halftone is influenced by the inks that are already printed. It is influenced by the underlying inks, but not by new ink layers printed on top of it.

The effective coverages are computed in the order in which they are printed. Assuming that the inks are printed in the order cyan, magenta, yellow, and black, we first compute the effective coverage of cyan. As no other ink is present when cyan is printed, the effective coverage of cyan is computed using the ink spreading curve of cyan alone, as in the single directive. Then, we compute the effective coverage of magenta. Since the cyan ink covers a percentage $c$ ' of the surface, a percentage $c^{\prime}$ of magenta is printed over solid cyan and a percentage (1-c') of magenta is printed alone on paper. We therefore use these percentages to weight the effective coverages returned by the ink spreading curve of magenta over solid cyan and of magenta alone. For yellow, we weight the ink spreading curves of yellow alone, yellow on cyan, yellow on magenta, and yellow on cyan and magenta according to the percentages of surface covered respectively by paper, (1-c') (1-m'); cyan only, $c^{\prime}\left(1-m^{\prime}\right)$; magenta only, (1-c') $m^{\prime}$; and cyan and magenta, $c^{\prime} m^{\prime}$.

We apply the same logic for the black ink halftone. We obtain a total of 15 ink spreading curves weighted according to the following equations:

$$
\begin{aligned}
& c^{\prime}=f_{c}(c) \\
& m^{\prime}=\left(1-c^{\prime}\right) \quad f_{m}(m) \\
& +\quad c^{\prime} \quad f_{m / c}(m) \\
& y^{\prime}=\left(1-c^{\prime}\right)\left(1-m^{\prime}\right) f_{y}(y) \\
& +\quad c^{\prime} \quad\left(1-m^{\prime}\right) f_{y / c}(y) \\
& +\left(1-c^{\prime}\right) \quad m^{\prime} \quad f_{y / m}(y) \\
& +\quad c^{\prime} \quad m^{\prime} \quad f_{y / c m}(y) \\
& \begin{array}{rcccl}
k^{\prime} & =\left(1-c^{\prime}\right) & \left(1-m^{\prime}\right) & \left(1-y^{\prime}\right) & f_{k}(k) \\
& +c^{\prime} & \left(1-m^{\prime}\right) & \left(1-y^{\prime}\right) & f_{k / c}(k) \\
& +\left(1-c^{\prime}\right) & m^{\prime} & \left(1-y^{\prime}\right) & f_{k / m}(k) \\
& +c^{\prime} & m^{\prime} & \left(1-y^{\prime}\right) & f_{k / c m}(k) \\
& +\left(1-c^{\prime}\right) & \left(1-m^{\prime}\right) & y^{\prime} & f_{k / y}(k) \\
& +c^{\prime} & \left(1-m^{\prime}\right) & y^{\prime} & f_{k / c y}(k) \\
& +\left(1-c^{\prime}\right) & m^{\prime} & y^{\prime} & f_{k / m y}(k) \\
& +c^{\prime} & m^{\prime} & y^{\prime} & f_{k / c m y}(k)
\end{array}
\end{aligned}
$$

The top directive improves the predictions, but due to the speed of printing devices, ink halftones that are already printed are not dry when the next layer is printed and may therefore be influenced by the newly printed ink halftone layer.

\section{c) Ink spreading of an ink halftone printed on top or below other ink layers}

Each ink halftone is influenced both by the inks already printed and by the inks that are printed on top of it. The ink spreading directive corresponding to this assumption is further referred to as the top or below directive.

As the top directive, the top or below directive weights the effective coverages returned by the different ink spreading curves according to the colorant surface coverages in the different superposition conditions. However, instead of considering only the inks already printed in the possible superposition conditions, the top or below directive accounts for all possible superposition conditions. Since, for 4 inks, there are 8 possible superposition conditions for each ink halftone, the effective coverage of an ink is the weighted average of 8 different ink spreading curves. For example, if a patch with 50\% cyan, 50\% magenta, and 50\% yellow is printed, the superposition dependent colorant surface coverages weighting the ink spreading curves of magenta are $25 \%$ unprinted paper, $25 \%$ cyan, $25 \%$ yellow, $25 \%$ green and $0 \%$ for the 4 superposition conditions containing solid black. For four inks, 32 ink spreading curves are required. 
Equations 5 weight the ink spreading curves of each ink halftone according to the corresponding colorant surface coverages. In these equations, computing the effective coverage of an ink requires the effective coverages of the other inks, which are not available. Because of the non-linearity of the equations, there is no analytical solution to the equation system. The effective coverages must therefore be computed iteratively, starting with the nominal coverages ${ }^{1}$. Usually, five iterations ensure sufficient convergence to determine the effective ink halftone coverages.

$$
\begin{aligned}
& c^{\prime}=\left(1-m^{\prime}\right)\left(1-y^{\prime}\right)\left(1-k^{\prime}\right) \quad f_{c}(c) \quad m^{\prime}=\left(1-c^{\prime}\right)\left(1-y^{\prime}\right) \quad\left(1-k^{\prime}\right) \quad f_{m}(m) \\
& +m^{\prime}\left(1-y^{\prime}\right)\left(1-k^{\prime}\right) f_{c / m}(c)+c^{\prime}\left(1-y^{\prime}\right)\left(1-k^{\prime}\right) f_{m / c}(m) \\
& +\left(1-m^{\prime}\right) \quad y^{\prime} \quad\left(1-k^{\prime}\right) f_{c / y}(c) \quad+\left(1-c^{\prime}\right) \quad y^{\prime} \quad\left(1-k^{\prime}\right) f_{m / y}(m) \\
& +m^{\prime} \quad y^{\prime}\left(1-k^{\prime}\right) f_{c / m y}(c)+c^{\prime} \quad y^{\prime}\left(1-k^{\prime}\right) f_{m / c y}(m) \\
& +\left(1-m^{\prime}\right)\left(1-y^{\prime}\right) \quad k^{\prime} \quad f_{c / k}(c) \quad+\left(1-c^{\prime}\right)\left(1-y^{\prime}\right) \quad k^{\prime} \quad f_{m / k}(m) \\
& +m^{\prime}\left(1-y^{\prime}\right) \quad k^{\prime} \quad f_{c / m k}(c)+c^{\prime}\left(1-y^{\prime}\right) \quad k^{\prime} \quad f_{m / c k}(m) \\
& +\left(1-m^{\prime}\right) \quad y^{\prime} \quad k^{\prime} \quad f_{c / y k}(c) \quad+\left(1-c^{\prime}\right) \quad y^{\prime} \quad k^{\prime} \quad f_{m / y k}(m) \\
& +m^{\prime} \quad y^{\prime} \quad k^{\prime} f_{c / m y k}(c)+c^{\prime} \quad y^{\prime} \quad k^{\prime} \quad f_{m / c y k}(m) \\
& y^{\prime}=\left(1-c^{\prime}\right)\left(1-m^{\prime}\right)\left(1-k^{\prime}\right) f_{y}(y) \quad k^{\prime}=\left(1-c^{\prime}\right)\left(1-m^{\prime}\right)\left(1-y^{\prime}\right) f_{k}(k) \\
& +\quad c^{\prime}\left(1-m^{\prime}\right)\left(1-k^{\prime}\right) f_{y / c}(y)+c^{\prime}\left(1-m^{\prime}\right)\left(1-y^{\prime}\right) f_{k / c}(k) \\
& +\left(1-c^{\prime}\right) \quad m^{\prime} \quad\left(1-k^{\prime}\right) f_{y / m}(y) \\
& +c^{\prime} \quad m^{\prime} \quad\left(1-k^{\prime}\right) f_{y / c m}(y) \\
& +\left(1-c^{\prime}\right)\left(1-m^{\prime}\right) \quad k^{\prime} \quad f_{y / k}(y) \\
& +\quad c^{\prime} \quad\left(1-m^{\prime}\right) \quad k^{\prime} \quad f_{y / c k}(y) \\
& +\left(1-c^{\prime}\right) \quad m^{\prime} \quad k^{\prime} \quad f_{y / m k}(y) \\
& +c^{\prime} \quad m^{\prime} \quad k^{\prime} \quad f_{y / c m k}(y) \\
& +\left(1-c^{\prime}\right) \quad m^{\prime} \quad\left(1-y^{\prime}\right) \quad f_{k / m}(k) \\
& +c^{\prime} \quad m^{\prime} \quad\left(1-y^{\prime}\right) f_{k / c m}(k) \\
& +\left(1-c^{\prime}\right)\left(1-m^{\prime}\right) \quad y^{\prime} \quad f_{k / y}(k) \\
& +\quad c^{\prime} \quad\left(1-m^{\prime}\right) \quad y^{\prime} \quad f_{k / c y}(k) \\
& +\left(1-c^{\prime}\right) \quad m^{\prime} \quad y^{\prime} \quad f_{k / m y}(k) \\
& +c^{\prime} \quad m^{\prime} \quad y^{\prime} \quad f_{k / c m y}(k)
\end{aligned}
$$

\section{RELEVANCE OF THE DIFFERENT INK SPREADING CURVES}

Using the top or below directive for the ink spreading model requires 32 different ink spreading curves. Creating these ink spreading curves requires at least 32 spectral measurements. In order to try to reduce the number of ink spreading curves, we evaluate their relevance. If a specific ink spreading curve does not improve the accuracy of the spectral prediction model, we try to discard it.

We use two different measurements set for the experiments. The first set, further referred to as the PIXMA set, is composed of the measurements of 625 patches printed on the Canon Pixma 4000 ink jet printer using classical rotated clustered dot screens at $100 \mathrm{lpi}$ and a resolution of $600 \mathrm{dpi}$. The 625 patches are composed of all combinations of cyan, magenta, yellow, and black at $0 \%, 25 \%, 50 \%, 75 \%$, and $100 \%$ nominal surface coverages.

The second set, further referred to as the CIEL set, is composed of the measurements of 750 patches printed on a newspaper web-offset press using classical rotated clustered dot screens at $75 \mathrm{lpi}$ and a resolution of $1200 \mathrm{dpi}$. The 750 patches are composed of all combinations of cyan, magenta, and yellow at $0 \%, 25 \%, 50 \%, 75 \%$, and $100 \%$ nominal surface coverages and black at $0 \%, 10 \%, 30 \%, 50 \%, 80 \%$, and $100 \%$.

The following experiment is performed both on the PIXMA and CIEL sets. It first calibrates the spectral prediction model, including the 32 ink spreading curves. Then, we successively modify each ink spreading curve by setting the effective coverage at $50 \%$ to a value between $0 \%$ and $100 \%$. For each modification, we predict the spectra of the patches in the measurement set and compute the average $\Delta \mathrm{E}_{94}$ between the measured and predicted spectra ${ }^{18}$. Each modification is therefore associated to an average $\Delta \mathrm{E}_{94}$. The resulting curves are called surface coverage accuracy curves.

In Appendix A, Figures 6 and 7 show the surface coverage accuracy curves for the PIXMA set (ink jet) and CIEL set (offset), respectively. The circles indicate the calibration points, i.e. prior to modifications, and the crosses indicate the effective coverages at $50 \%$ yielding the minimum average $\Delta \mathrm{E}_{94}$. All the surface coverage accuracy curves have the shape of convex parabolas, but not all parabolas have similar curvatures. Some are nearly flat, especially the ink spreading 
curves whose superposition condition includes solid black, i.e. the last four of the first three lines of the surface coverage accuracy curves. We further refer to these 12 ink spreading curves as the ink spreading curves on solid black.

When observing the surface coverage accuracy of ink spreading curves which do not include solid black, we make the following observations. The effective coverages at 50\% chosen during calibration (circles) agree well with the minima (crosses). Moreover, the surface coverage accuracy curves are highly relevant to the accuracy of the prediction model because of their curvature. If the effective coverage at $50 \%$ of one of these ink spreading curves is set too far off the minimum, the average $\Delta \mathrm{E}_{94}$ becomes large. Finally, we can see that the effective coverage at $50 \%$ of a given ink halftone yielding the minimum $\Delta \mathrm{E}_{94}$ is different depending on the superposition condition. This emphasizes the fact that the ink spreading of an ink halftone depends on the presence of other inks.

The opposite analysis is true for the ink spreading curves on solid black. The calibrated effective surface coverages do not agree well with the computed minima. They are not relevant for the accuracy of the prediction model because the curvature of the parabolas is low, i.e. a modification of an effective halftone surface coverage at $50 \%$ nominal coverage on solid black does not deteriorate the accuracy of the prediction model. The system is underdetermined ${ }^{3}$ and it seems preferable to discard the ink spreading curves on solid black.

\section{RESILIENCE OF THE INK SPREADING CURVES TO NOISE}

The ink spreading curves on black do not improve the accuracy of the spectral prediction model. Let us further show that they are not only irrelevant, but also highly subject to noise. There are indeed several possible origins of noise in the measured spectra. Noise comes from the printing device itself, from dirty patches, from variations in the intrinsic reflectance of the substrate, from variations in the printing conditions, etc. These different sources produce each a different kind of noise, making them difficult to separate and model. We therefore use Gaussian noise and analyze its impact on the calibration of the ink spreading curves.

In the following experiment, we observe how stable the calibration of the ink spreading curves is in the presence of Gaussian noise added to each wavelength of the measured reflectance with both average and standard deviation between 0 and 0.05 . For each ink spreading curve and each noise average, we calibrate 500 times the effective coverage of the $50 \%$ patch as follows: We first choose randomly the standard deviation of the noise between 0 and 0.05 . We generate the noise independently for each wavelength and add it to the measured spectrum of the $50 \%$ patch. The ink spreading curve is established using this measured noise augmented reflectance spectrum. For each ink spreading curve and each noise average, we have 500 different fitted effective coverages.

The results are shown in Appendix B. Figures 8 and 9 show the results for the PIXMA (ink jet) and CIEL (offset) measurement sets, respectively. Both figures present the same behavior: The effective surface coverages superposed with solid black are very sensitive to noise, i.e. the same noise induces very large effective surface coverage calibration variations. On the other hand, the other effective surface coverages are mainly subject to the mean value of the Gaussian noise. As expected, when adding noise with a positive mean, the predicted reflection spectra become higher. This positive difference is compensated by lower effective surface coverages.

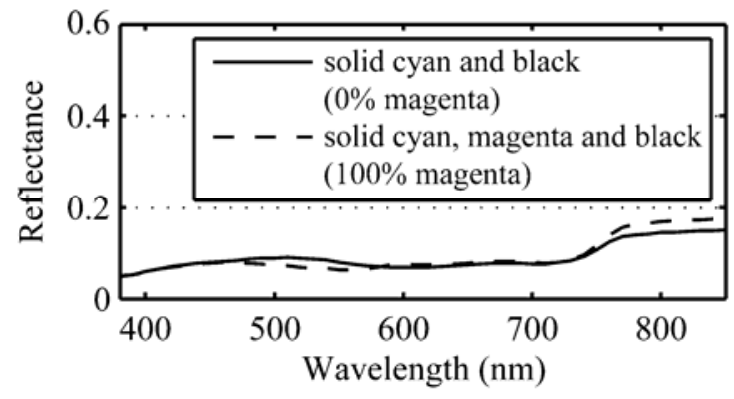

Figure 3. Spectra of solid cyan supposed with solid black (continuous line) and of solid cyan superposed with solid magenta and black (dashed line) from the CIEL measurement set.

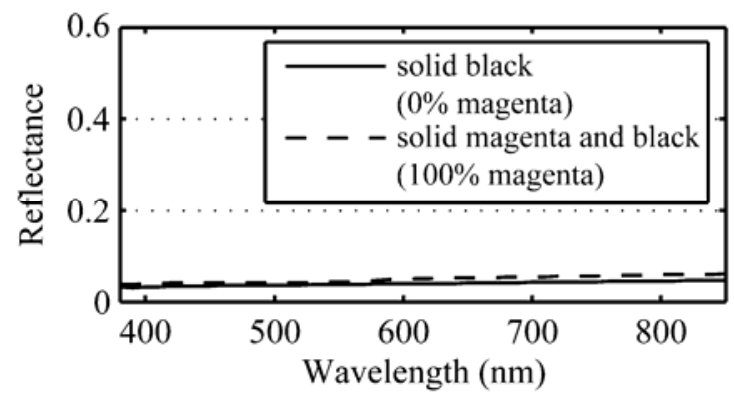

Figure 4. Spectra of solid black (continuous line) and of solid magenta superposed with solid black (dashed line) from the PIXMA measurement set.

The problem caused by the ink spreading curves on solid black is underlined in Figures 3 and 4. In Figure 3, we focus on the magenta ink spreading curve over solid cyan and solid black for the CIEL measurement set. The reflection spectrum without magenta corresponds to the beginning of the ink spreading curve ( $0 \%$ coverage of magenta). The spectrum with 
solid magenta corresponds to the end of the ink spreading curve (100\% coverage of magenta). We see that the difference between the two spectra is negligible. Therefore, the ink spreading curve of magenta over solid cyan and black has a negligible influence when predicting reflection spectra. Moreover, noise has a large impact on the calibration of the ink spreading curve because small variations in the 50\% magenta coverage spectrum must be compensated by large variations in the ink spreading curve.

The same reasoning applies to Figure 4 when considering the ink spreading curve of magenta over solid black (PIXMA set). The result of the calibration of the ink spreading curves for the PIXMA measurement set is shown in Figure 5. Figure 5 shows the dot gain curves, defined as effective minus nominal surface coverage in function of nominal surface coverage. All the dot gain curves except those on solid black show a consistent behavior. Conversely, we can see the effect of measurement noise on the dot gain curves superposed with solid black: The curves suddenly behave randomly.

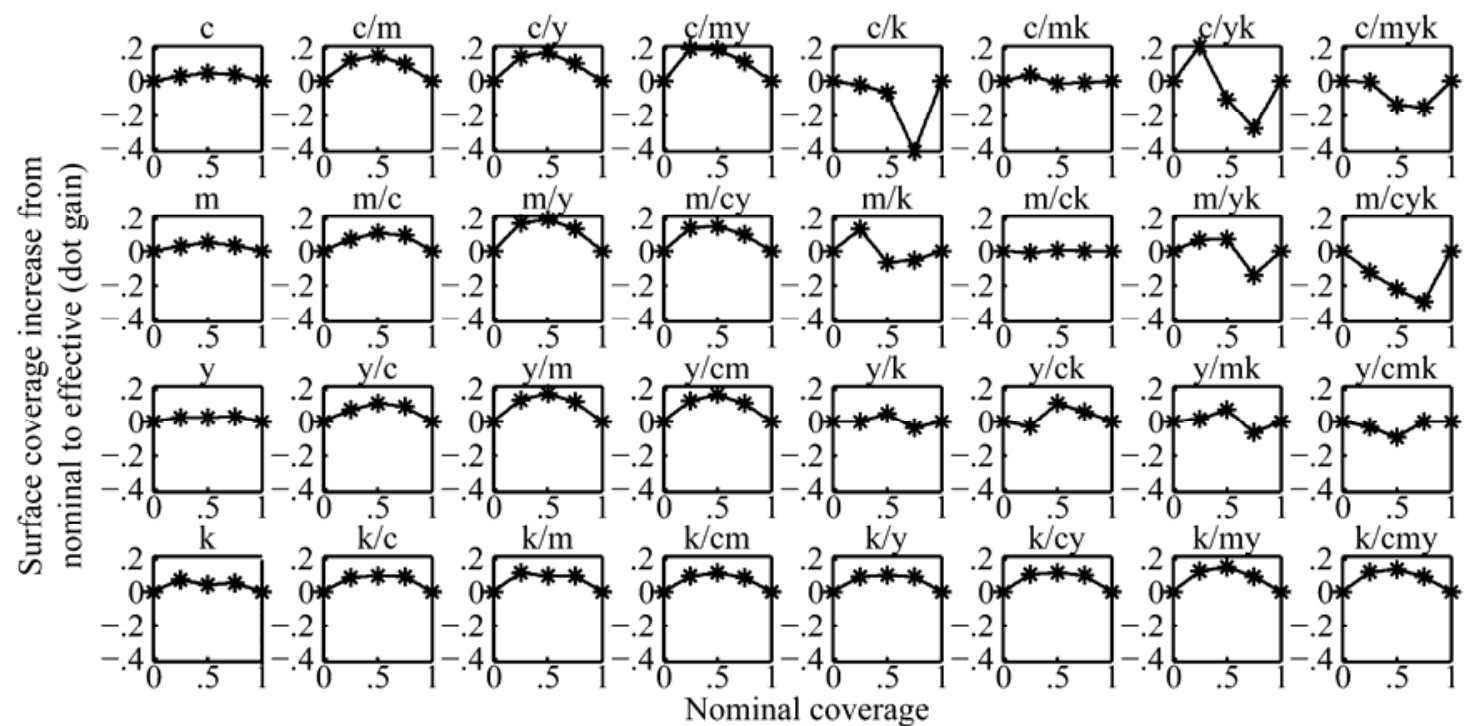

Figure 5. Dot gain curves, derived from the corresponding ink spreading curves, calibrated at $25 \%, 50 \%$, and $75 \%$ nominal coverage on the PIXMA measurement set. The horizontal axis corresponds to the nominal surface coverage of the ink halftone and the vertical axis to the dot gain.

\section{REMOVING THE INK SPREADING CURVES ON SOLID BLACK}

In the previous two sections, we have shown that the ink spreading curves on solid black are not resilient to noise and do not improve the accuracy of the spectral prediction model. We therefore propose a new directive for the ink spreading model called halftone black that discards these 12 ink spreading curves, yielding the following set of equations:

$$
\begin{aligned}
& c^{\prime}=\left(1-m^{\prime}\right)\left(1-y^{\prime}\right) \quad f_{c}(c) \quad m^{\prime}=\left(1-c^{\prime}\right)\left(1-y^{\prime}\right) \quad f_{m}(m) \\
& +m^{\prime}\left(1-y^{\prime}\right) f_{c / m}(c)+c^{\prime}\left(1-y^{\prime}\right) f_{m / c}(m) \\
& +\left(1-m^{\prime}\right) \quad y^{\prime} \quad f_{c / y}(c)+\left(1-c^{\prime}\right) \quad y^{\prime} \quad f_{m / y}(m) \\
& +m^{\prime} \quad y^{\prime} \quad f_{c / m y}(c)+c^{\prime} \quad y^{\prime} \quad f_{m / c y}(m) \\
& y^{\prime}=\left(1-c^{\prime}\right)\left(1-m^{\prime}\right) \quad f_{y}(y) \quad k^{\prime}=\left(1-c^{\prime}\right)\left(1-m^{\prime}\right)\left(1-y^{\prime}\right) \\
& +c^{\prime}\left(1-m^{\prime}\right) f_{y / c}(y)+c^{\prime}\left(1-m^{\prime}\right)\left(1-y^{\prime}\right) f_{k / c}(k) \\
& +\left(1-c^{\prime}\right) \quad m^{\prime} \quad f_{y / m}(y)+\left(1-c^{\prime}\right) \quad m^{\prime} \quad\left(1-y^{\prime}\right) f_{k / m}(k) \\
& +c^{\prime} \quad m^{\prime} \quad f_{y / c m}(y)+c^{\prime} \quad m^{\prime}\left(1-y^{\prime}\right) f_{k / c m}(k) \\
& +\left(1-c^{\prime}\right)\left(1-m^{\prime}\right) \quad y^{\prime} \quad f_{k / y}(k) \\
& +c^{\prime}\left(1-m^{\prime}\right) \quad y^{\prime} \quad f_{k / c y}(k) \\
& +\left(1-c^{\prime}\right) \quad m^{\prime} \quad y^{\prime} \quad f_{k / m y}(k) \\
& +c^{\prime} \quad m^{\prime} \quad y^{\prime} \quad f_{k / c m y}(k)
\end{aligned}
$$


Using the halftone black directive, it is possible to break the computation of the effective coverages into two steps: First, we compute the effective coverages for cyan, magenta, and yellow using the iterative method proposed for the top or below directive. Then, we directly compute the black effective coverage using the effective coverages of the other inks.

The accuracy of the different ink spreading directives proposed in Section 3.2 and Section 6 are compared in Table 2 for the PIXMA measurement set and in Table 3 for the CIEL measurement set. On the PIXMA measurement set, the halftone black directive yields the best accuracy while the top or below directive suffers from the measurement noise as seen in Figure 5. On the CIEL measurement set, both directives yield similar results. Considering that the patches are printed on newsprint paper, which is non-coated and less uniform than traditional paper, the achieved accuracy is remarkable. We still recommend using halftone black. It uses only 20 ink spreading curves instead of 32, reduces the calibration effort and decreases the time required to perform a spectral prediction.

Table 2. Accuracy of the spectral prediction model on the PIXMA measurement set. For each of the 625 patches of the measurement set, we compute the $\Delta \mathrm{E}_{94}$ between the predicted and measured spectra. The statistics show the average $\Delta \mathrm{E}_{94}$, the corresponding standard deviation, $95 \%$ percentile, and maximum $\Delta \mathrm{E}_{94}$. The Yule-Nielsen factor is $n=10$.

\begin{tabular}{|l|rrrr|}
\hline & Average & St. Deviation & 95\% percentile & Maximum \\
\hline No ink spreading & 4.29 & 2.70 & 8.68 & 11.90 \\
Single & 3.16 & 2.00 & 6.31 & 9.62 \\
Top & 2.28 & 1.69 & 5.50 & 8.32 \\
Top or below & 2.10 & 1.40 & 4.59 & 7.10 \\
Halftone black & 1.58 & 1.08 & 3.57 & 5.65 \\
\hline
\end{tabular}

Table 3. Accuracy of the spectral prediction model on the CIEL measurement set. For each of the 750 patches of the measurement set, we compute the $\Delta \mathrm{E}_{94}$ between the predicted and measured spectra. The statistics show the average $\Delta \mathrm{E}_{94}$, the corresponding standard deviation, $95 \%$ percentile, and maximum $\Delta \mathrm{E}_{94}$. The Yule-Nielsen factor is $n=7.8$.

\begin{tabular}{|l|rrrr|}
\hline & Average & St. Deviation & 95\% percentile & Maximum \\
\hline No ink spreading & 4.76 & 2.27 & 8.13 & 10.38 \\
Single & 2.20 & 1.11 & 4.03 & 5.27 \\
Top & 1.46 & 0.83 & 3.15 & 4.01 \\
Top or below & 1.47 & 0.82 & 3.03 & 3.99 \\
Halftone black & 1.44 & 0.83 & 3.08 & 3.98 \\
\hline
\end{tabular}

\section{CONCLUSION}

After introducing the Yule-Nielsen modified Spectral Neugebauer prediction model, we present the ink spreading model and its different directives. We then perform experiments to deduce the ink spreading curves that have a high impact on the accuracy of the spectral prediction model and that are resilient to measurement noise. Based on this in-depth analysis, we show that the ink spreading curves of ink halftones superposed with solid black are neither relevant for the accuracy of the spectral prediction model nor resilient to measurement noise. We therefore propose an improved ink spreading directive called halftone black relying on a reduced set of ink spreading equations.

This new directive is compared with the other ones for two different measurement sets, one from a consumer-grade inkjet printer and the other from a newspaper web-offset press. In both cases the halftone black directive performs best on average. Depending on the printing technology, the other directives may sometimes show similar performances, but since halftone black requires only 20 ink spreading curves instead of 32, we recommend it for CMYK spectral prediction models.

\section{ACKNOWLEDGMENT}

Part of this research was performed within the framework of the CTI project 6498.1 supported by the Swiss government agency for the promotion of innovation. The project has also been partially supported by the Swiss National Science Foundation, grant $n^{\circ} 105119$. 


\section{REFERENCES}

1 R. D. Hersch, P. Emmel, F. Collaud, F. Crété, "Spectral reflection and dot surface prediction models for color halftone prints", Journal of Electronic Imaging, Vol. 14, No. 3, 33001-12 (2005)

2 R. D. Hersch, F. Crété, "Improving the Yule-Nielsen modified spectral Neugebauer model by dot surface coverages depending on the ink superposition conditions", Color Imaging X: Processing, Hardcopy, and Applications, SPIE Vol. 5667, 434-445 (2005)

3 Th. Bugnon, M. Brichon and R.D. Hersch, "Model-Based Deduction of CMYK Surface Coverages from Visible and Infrared Spectral Measurements of Halftone Prints", Color Imaging XII: Processing, Hardcopy, and Applications, SPIE Vol. 6493, 649310-1 to 649310-10 (2007);

4 D.R.Wyble, R.S. Berns, "A Critical Review of Spectral Models Applied to Binary Color Printing”, Journal of Color Research and Application, Vol. 25, No. 1, 4-19 (2000)

5 R. Balasubramanian, "Optimization of the spectral Neugebauer model for printer characterization", Journal of Electronic Imaging, Vol. 8, No. 2, 156-166 (1999)

6 T. Ogasahara, "Verification of the Predicting Model and Characteristics of Dye-Based Ink Jet Printer", Journal of Imaging Science and Technology, Vol. 48, No. 2, 130-137 (2004)

7 J.A.C. Yule, W.J. Nielsen, "The penetration of light into paper and its effect on halftone reproductions", Proc. TAGA Vol. 3, 65-76 (1951)

8 J.A.S Viggiano, "Modeling the Color of Multi-Colored Halftones", Proc. TAGA, 44-62 (1990)

9 H.E.J. Neugebauer, "Die theoretischen Grundlagen des Mehrfarbendrucks", Zeitschrift fuer wissenschaftliche Photographie, Vol. 36, 36-73 (1937), reprinted in Neugebauer Seminar on Color Reproduction, SPIE Vol. 1184, 194202 (1989)

10 M.E. Demichel, Procédé, Vol. 26, 17-21 (1924)

11 A. Murray, "Monochrome reproduction in photoengraving", J. Franklin Institute, Vol. 221, 721-724 (1936)

12 H.R. Kang, "Applications of color mixing models to electronic printing", Journal of Electronic Imaging, Vol. 3, No. 3, 276-287 (1994)

13 R.D. Hersch, F. Collaud, P. Emmel, "Reproducing color images with embedded metallic patterns", Proc. SIGGRAPH 2003, ACM Trans. on Graphics, Vol. 22, No. 3, 427-436 (2003)

14 K. Iino, R.S. Berns, "Building color management modules using linear optimization I. Desktop", Journal of Imaging Science and Technology, Vol. 42, No. 1, 79-94 (1998)

15 K. Iino, R.S. Berns, "Building color management modules using linear optimization II. Prepress system for offset printing", Journal of Imaging Science and Technology, Vol. 42, No. 2, 99-114 (1998)

16 A. U. Agar and J. P. Allebach, "An Iterative Cellular YNSN Method for Color Printer Calibration", Proc. of the 6th IS\&T/SID Color Imaging Conference, Scottsdale AZ, 197-200 (1998)

17 S. Zuffi, R. Schettini, "An innovative method for spectral-based printer characterization", Color Imaging: DeviceIndependent Color, Color Hardcopy, and Applications VII (R. Eschbach, G.G. Marcu eds.), SPIE Vol. 4663, 1-7 (2002)

18 G. Sharma, "Digital Color Imaging Handbook", Chapter 1, 35-36, CRC Press, 2003

Proc. SPIE Vol. 6807, 680717, Color Imaging XIII: Processing, Hardcopy, and Applications; Reiner Eschbach, Gabriel G. Marcu, Shoji Tominaga; Eds., January 2008 


\section{APPENDIX A}

The following two figures show the impact of the ink spreading curves on the prediction accuracy of the EYNSN model. For each ink spreading curve, we modify the effective coverage at $50 \%$ nominal coverage and compute the average $\Delta \mathrm{E}_{94}$ between the measured and predicted spectra. The circle indicates the effective coverage at $50 \%$ nominal coverage deduced during calibration. The cross indicates the effective coverage that minimizes the average $\Delta \mathrm{E}_{94}$. The experiment is performed once for an inkjet printer and once for an offset press.

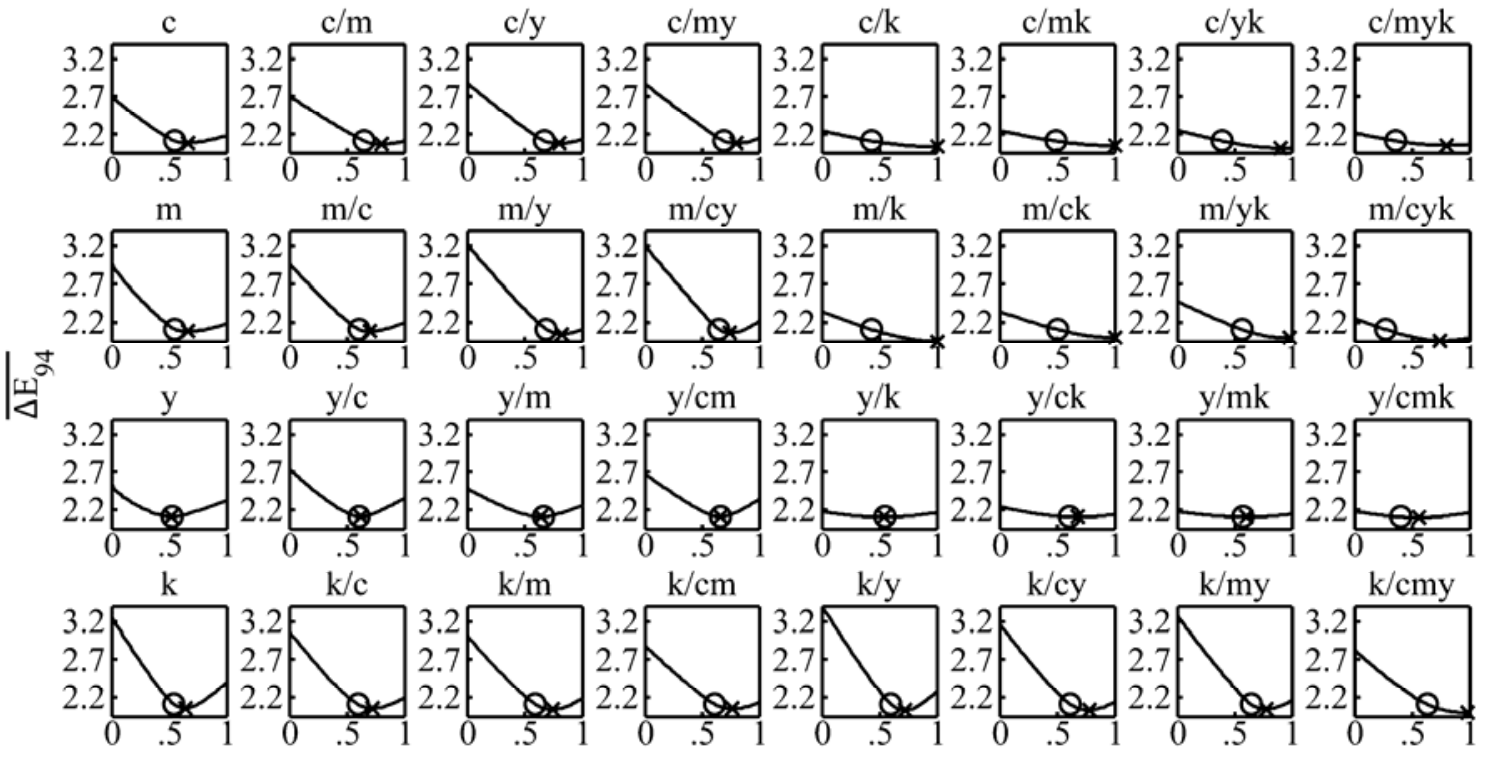

Effective coverage at $50 \%$ nominal coverage for the specified dot gain curve

Figure 6. Surface coverage accuracy curves showing the impact of dot gain variations computed for the PIXMA measurement set (inkjet, $100 \mathrm{lpi}$, Yule-Nielsen factor $n=10$ ). The horizontal axis denotes the effective surface coverage at $50 \%$ nominal surface coverage and the vertical axis the prediction accuracy in terms of average $\Delta \mathrm{E}_{94}$ between measured and predicted spectra.
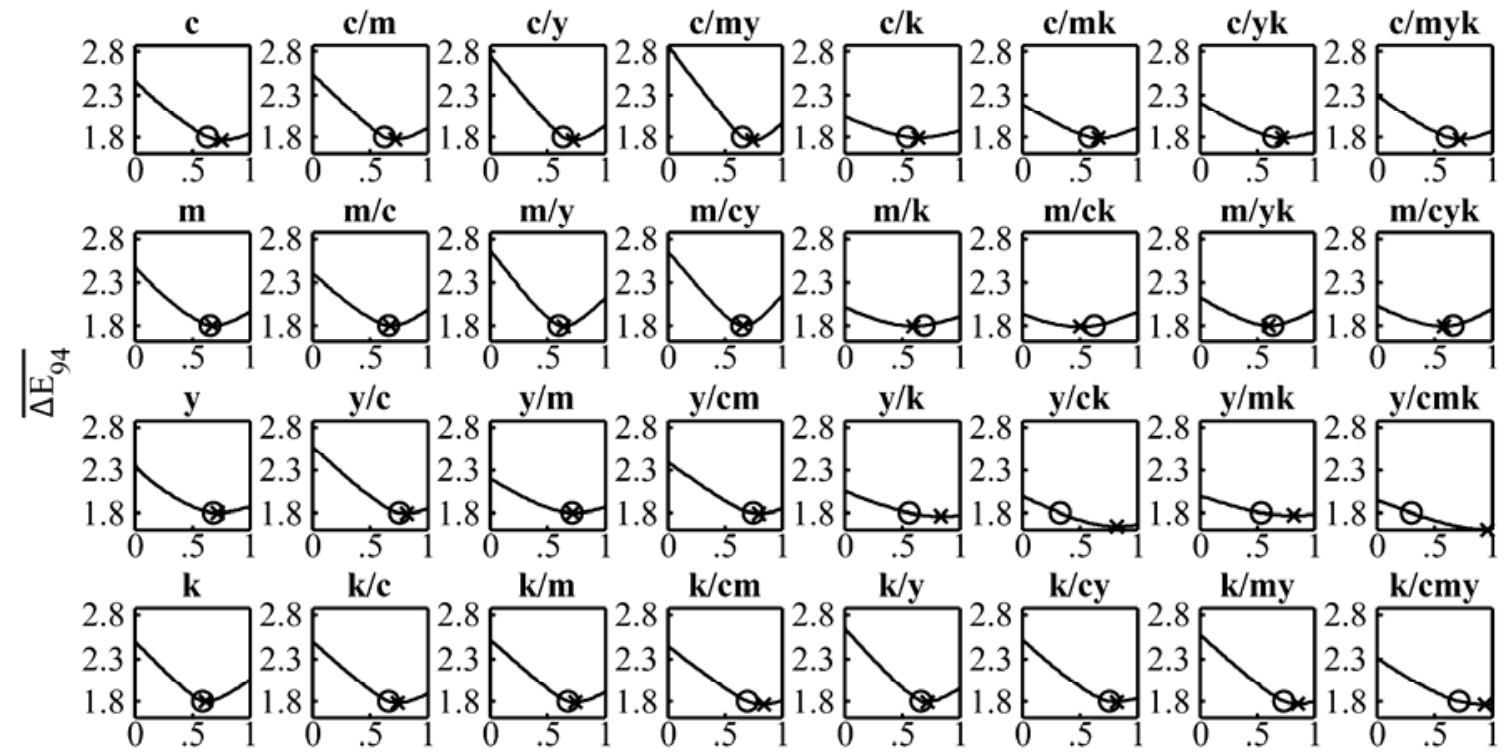

Effective coverage at $50 \%$ nominal coverage for the specified dot gain curve

Figure 7. Surface coverage accuracy curves showing the impact of dot gain variations computed on the CIEL measurement set (offset, $150 \mathrm{lpi}$, Yule-Nielsen factor $n=7.8$ ). The horizontal axis denotes the effective surface coverage at $50 \%$ nominal surface coverage and the vertical axis the prediction accuracy in terms of average $\Delta \mathrm{E}_{94}$ between measured and predicted spectra. 


\section{APPENDIX B}

The following two figures show the impact of noise on the calibration of the ink spreading curves. For each ink spreading curve and each noise average, we calibrate 500 times the $50 \%$ patch as follows: We first choose randomly the standard deviation of the noise between 0 and 0.05 . Then, we generate the noise independently for each wavelength and add it to the measured spectrum of the $50 \%$ patch. Finally, we calibrate the ink spreading curve using this noisy spectrum. For each ink spreading curve and each noise average, we therefore have 500 different fitted effective coverages. In the figures, we plot the average and standard deviations of the fitted effective coverages. The dotted line represents the calibration without noise. The Yule-Nielsen factor is $n=7.8$ for the CIEL set and $n=10$ for the PIXMA set.

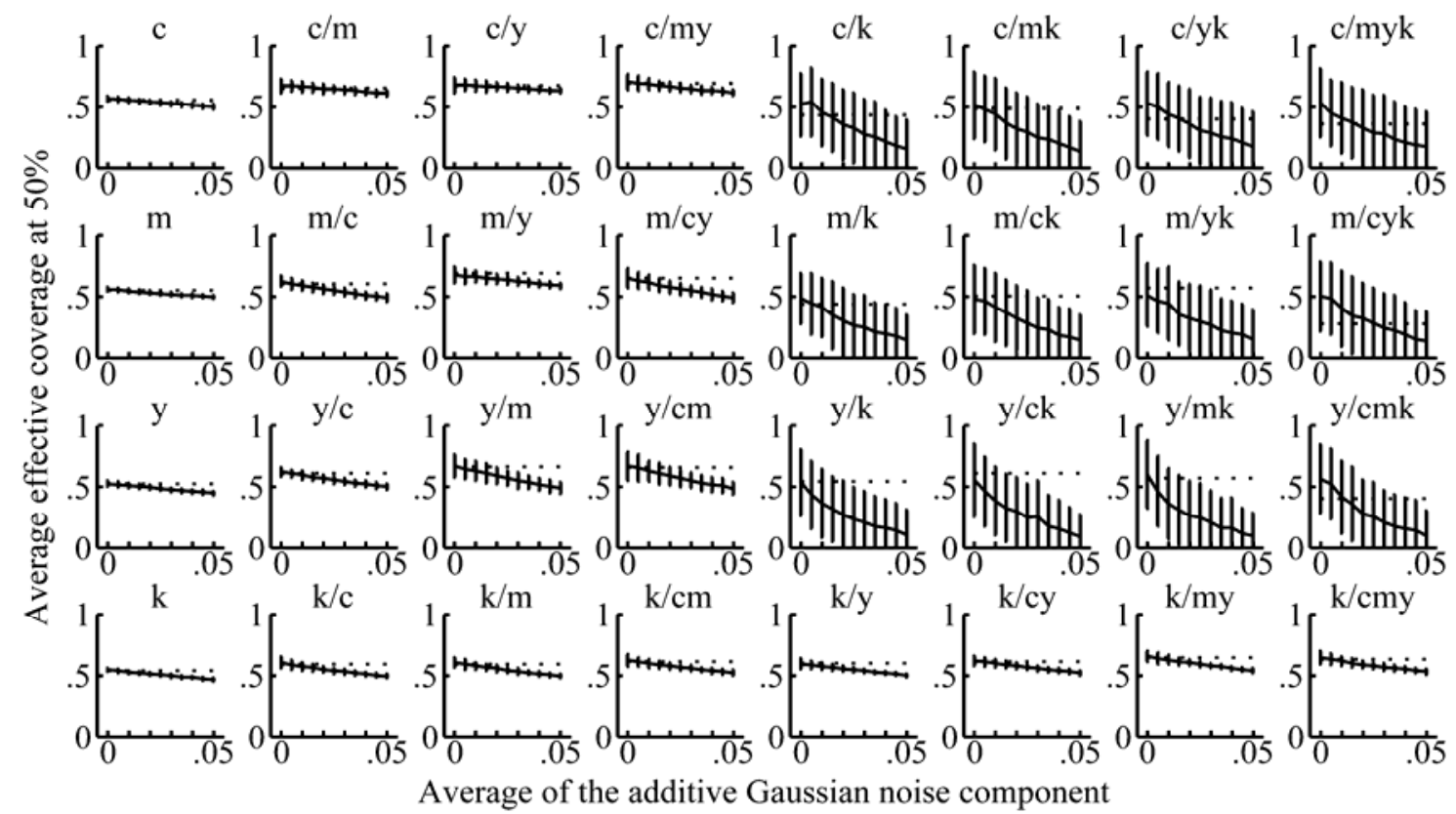

Figure 8. Impact of noise on the calibration of the ink spreading curves computed for the PIXMA measurement set.

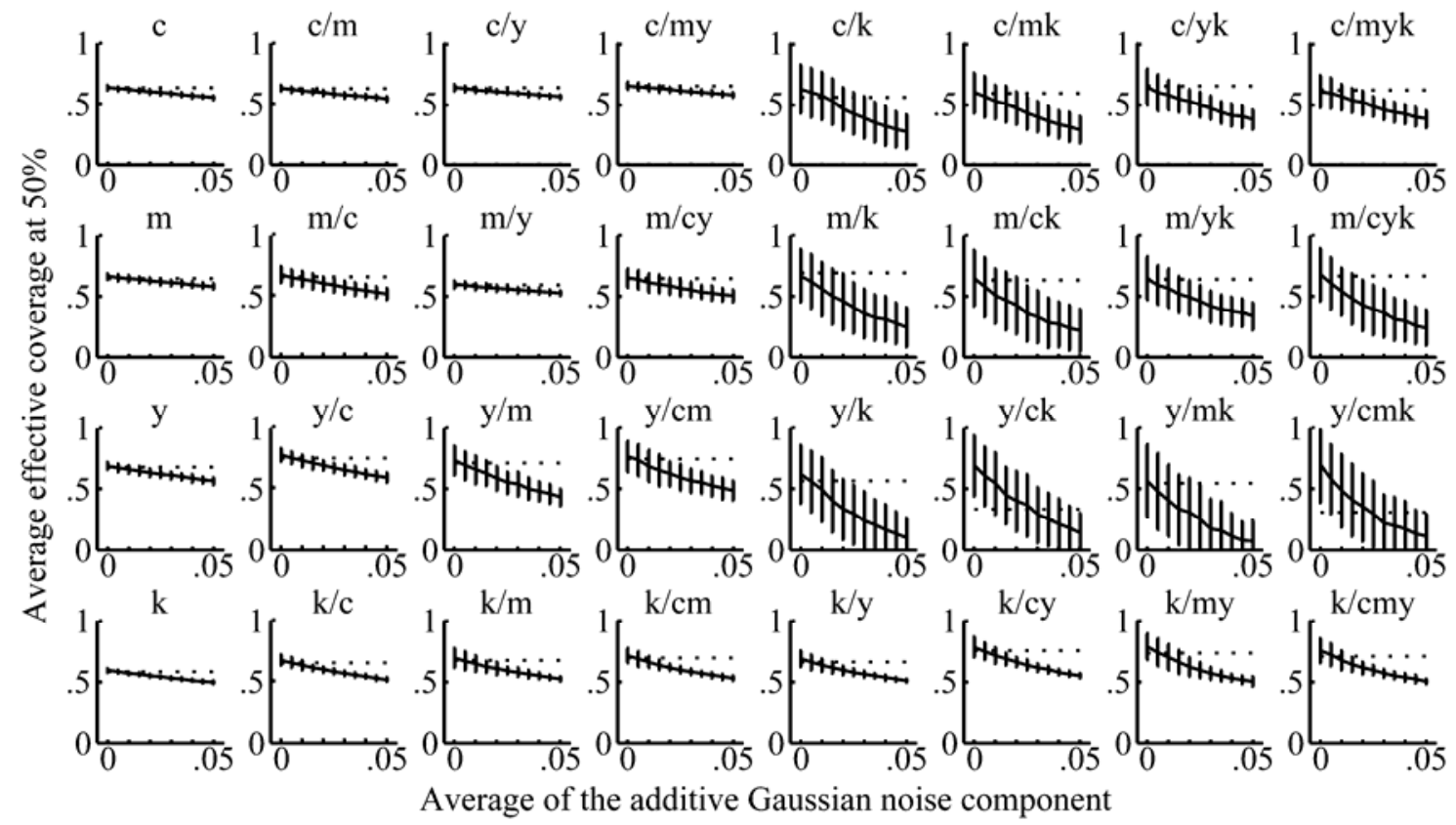

Figure 9. Impact of noise on the calibration of the ink spreading curves computed for the CIEL measurement set. 